\title{
Extensa epúlide congênita em uma recém-nascida: diagnóstico e tratamento de uma
}

\section{lesão rara}

\author{
Extensive congenital epulis in a newborn: diagnosis and treatment of a rare lesion \\ Épulis congénito extensivo en un recién nacido: diagnóstico y tratamiento de una lesión rara
}

Recebido: 25/01/2021 | Revisado: 26/01/2021 | Aceito: 27/01/2021 | Publicado: 04/02/2021

Ciro Borges Duailibe de Deus

ORCID: https://orcid.org/0000-0001-9684-6956 Universidade Estadual Paulista "Júlio de Mesquita Filho", Brasil Universidade Estácio de Sá, Brasil Faculdade Pitágoras, Brasil

E-mail: ciro_duailibe@hotmail.com

João Victor Uchôa Silva

ORCID: https://orcid.org/0000-0003-0840-5902 Faculdade Pitágoras, Brasil

E-mail: joaovictorneto10@hotmail.com

André Hergesel de Oliva

ORCID: https://orcid.org/0000-0001-5818-9350

Universidade Estadual Paulista "Júlio de Mesquita Filho", Brasil

E-mail: holivaandre@gmail.com

Wellington José Alves Nunes

ORCID: https://orcid.org/0000-0002-4846-132X Serviço Especializado de Odontologia, Brasil Instituto de Odontologia do Maranhão, Brasil E-mail: wjanunes@globo.com

Roberta Okamoto

ORCID: https://orcid.org/0000-0002-6773-6966 Universidade Estadual Paulista “Júlio de Mesquita Filho", Brasil E-mail: beta.okamoto@gmail.com

\begin{abstract}
Resumo
A epúlide congênita é um tumor benigno e raro que acomete a mucosa oral de recém-nascidos. Apesar de ser benigna, esta lesão pode atingir extensa dimensão e prejudicar a função do complexo maxilomandibular tanto para alimentação, quanto para respiração, devendo então ser diagnosticada o mais precocemente possível para que seja realizada sua remoção. O presente artigo tem como objetivo relatar um caso de diagnóstico e tratamento de uma recém-nascida com epúlide congênita de grandes proporções. Paciente M.C.A., leucoderma, 2,7kg, gênero feminino e normorreativa, apresentava no exame físico do período pós-natal imediato, uma lesão nodular, lisa, não-ulcerada e pediculada com 40 milímetros em seu maior diâmetro, localizada em mucosa alveolar vestibular de maxila direita. Com a integração multiprofissional entre a equipe médica de pediatria, radiológica e cirurgia buco-maxilo-facial, foram solicitados e avaliados os exames de tomografia computadorizada e ultrassonografia com doppler para avaliação do fluxo sanguíneo da lesão e exclusão definitiva do diagnóstico diferencial de hemangioma. O procedimento de exérese foi realizado sem intercorrências sob anestesia geral, quando o paciente datava onze dias de vida. A peça anatômica foi enviada para avaliação histopatológica e imuno-histoquímica, que confirmaram o diagnóstico clínico de epúlide congênita. A paciente apresentou recuperação nutricional imediata e evoluiu satisfatoriamente. A epúlide congênita por se tratar de uma condição rara, acaba sendo muitas vezes subdiagnosticada e confundida com lesões vasculares, por isso, a abordagem multiprofissional direciona o diagnóstico e o tratamento de forma adequada.
\end{abstract}

Palavras-chave: Epúlide; Hemangioma; Ultrassonografia.

\begin{abstract}
Congenital epulis is a rare benign tumor that affects the oral mucosa of newborns. Despite being benign, this lesion can reach an extensive dimension and impair the function of the maxillomandibular complex for both feeding and breathing, and should therefore be diagnosed as early as possible to perform its excision. This article aims to report a case of diagnosis and treatment of a newborn with large congenital epulis. Patient M.C.A., leucoderma, $2.7 \mathrm{~kg}$, female and normorreative, presented in the physical examination of the immediate postnatal period, a nodular, smooth, nonulcerated and pediculated lesion with 40 millimeters in its largest diameter, located in the buccal alveolar mucosa of right jaw. With the multiprofessional integration between the pediatric, radiological and buccomaxillofacial medical team, computed tomography and doppler ultrasonography exams were requested and evaluated to assess the blood
\end{abstract}


flow of the lesion and definitive exclusion of the differential diagnosis of hemangioma. The excision procedure was performed under general anesthesia, when the patient was eleven days old, uneventful and the anatomical specimen was stored for histopathological and immunohistochemical evaluation, which confirmed the clinical diagnosis of congenital epulis. The patient showed immediate nutritional recovery and progressed satisfactorily. The congenital epulis, being a rare condition, ends up being underdiagnosed and confused with vascular lesions, therefore, the multiprofessional approach directs the diagnosis and treatment in an appropriate way.

Keywords: Epulis; Hemangioma; Ultrasonography.

\section{Resumen}

El épulis congénito es un tumor benigno poco común que afecta la mucosa oral de los recién nacidos. A pesar de ser benigna, esta lesión puede alcanzar una dimensión extensa y perjudicar la función del complejo maxilomandibular tanto para la alimentación como para la respiración, por lo que debe diagnosticarse lo antes posible para realizar su escisión. Este artículo tiene como objetivo reportar un caso de diagnóstico y tratamiento de un recién nacido con épulis congénito grande. Paciente M.C.A., leucodermia, $2,7 \mathrm{~kg}$, del sexo femenino y normorreativo, que presentó en la exploración física del puerperio inmediato una lesión nodular, lisa, no ulcerada y pediculada de 40 mm en su diámetro mayor, localizada en la mucosa alveolar bucal de mandíbula derecha. Con la integración multiprofesional entre el equipo médico pediátrico, radiológico y buccomaxilofacial, se solicitaron y evaluaron exámenes de tomografía computarizada y ecografía doppler para valorar el flujo sanguíneo de la lesión y exclusión definitiva del diagnóstico diferencial de hemangioma. El procedimiento de exéresis se realizó bajo anestesia general, cuando la paciente cumplió once días, sin incidentes y la pieza anatómica fue almacenada para evaluación histopatológica e inmunohistoquímica, que confirmó el diagnóstico clínico de épulis congénito. El paciente mostró una recuperación nutricional inmediata y una evolución satisfactoria. El épulis congénito, al ser una condición poco frecuente, acaba infradiagnosticarse y se confunde con las lesiones vasculares, por lo que el abordaje multiprofesional orienta el diagnóstico y tratamiento de forma adecuada.

Palabras clave: Epulis; Hemangioma; Ultrasonografía.

\section{Introdução}

A epúlide congênita (EC) é uma lesão que acomete exclusivamente fetos e recém-nascidos (RN), caracterizada como um tumor raro, benigno e de etiologia não completamente compreendida. Composta por células granulares, também conhecida como epúlide granular congênita, tumor congênito de células granulares e tumor de Neumann's (Elías Podestá, Cóndor Astucuri, García Gutiérrez, Arellano Sacramento, \& Elías Díaz, 2017; Jain, Sinha, \& Singh, 2020; Lee, Kim, \& Shin, 2013; Marinho, Santos, Morais, \& Freitas, 2020; Rahman, Ismail, Mohamad, Yusof, \& Mohamad, 2015; Rodrigues et al., 2019).

Este tumor possui superfície lisa, de coloração que varia de rosada a avermelhada, de acordo com a sua perfusão sanguínea, surgindo diretamente da mucosa alveolar. Esta afecção é revelada circunscrita, firme, séssil ou pediculada, sem ulcerações, podendo ainda ser encontrada uni ou multilobuladas, com uma ampla variação de tamanho, se apresentando contido na cavidade oral, quando de pequenas dimensões, ou, projetando-se para o exterior da boca quando em situações extremas (Cheung \& Putra, 2020; Elías Podestá et al., 2017).

Estas lesões geralmente surgem a partir das cristas alveolares, são solitárias, mas em alguns casos mais de um tumor pode ser identificado, tendo ainda predileção pelo gênero feminino. Possui maior prevalência de acometimento na região anterior da maxila, o que, dependendo de sua extensão, poderá ocasionar dificuldade de alimentação, obstrução das vias aéreas e grande comprometimento estético do recém-nascido (de Paulo, da Cruz Perez, Rosa, \& Durighetto Junior, 2013; Lee et al., 2013; Rahman et al., 2015). A EC apresenta o seu desenvolvimento interrompido após o nascimento, o que sugere íntima relação com as alterações hormonais da gravidez (Cheung \& Putra, 2020).

Histologicamente este tumor se encontra encapsulado, com proliferação de células de morfologia poligonal, núcleos excêntricos ovais e citoplasma eosinofílico granuloso, envolvido por um epitélio estratificado contido, sem projeções exteriores (Cheung \& Putra, 2020; Elías Podestá et al., 2017).

A abordagem multidisciplinar envolvendo pediatra, cirurgião buco-maxilo-facial, anestesiologista e patologista é obrigatória para uma condução adequada dessas lesões. (Lee et al., 2013). 
O objetivo deste trabalho é relatar através de um caso clínico, as características clínicas e histológicas, assim como, realizar o diagnóstico diferencial e manejo cirúrgico da epúlide congênita em uma recém-nascida de 11 dias de vida.

\section{Metodologia}

Trata-se de um relato de caso clínico cirúrgico, que consiste em um tipo de estudo descritivo e retrospectivo, realizado por meio da técnica de observação direta e revisão de literatura sobre o tema através de artigos encontrados nas bases de dados SCIELO (Scientific Eletronic Library On-line), PubMed, Science Direct e Google Acadêmico. Os responsáveis pela paciente autorizaram a divulgação dos dados e exibição das imagens por meio de assinatura de Termo de Consentimento Livre e Esclarecido (TCLE), respeitando todos os princípios éticos da Declaração de Helsinque.

\section{Relato de Caso}

A RN M.C.A., leucoderma, nascida a termo através de parto normal com $2.875 \mathrm{~g}$, sem comorbidades sistêmicas, cursava com lesão hiperplásica pediculada, não ulcerada, lisa e avermelhada, consistente à palpação, de grandes proporções 40x20x20mm, em região de mucosa alveolar vestibular de maxila lado direito, o que dificultava a pega do peito e causava irritação, comprometendo a sua nutrição adequada (Figuras 1A e B). Esta lesão foi detectada no período pós natal imediato.

Figuras 1A e B - Aspecto clínico da lesão.

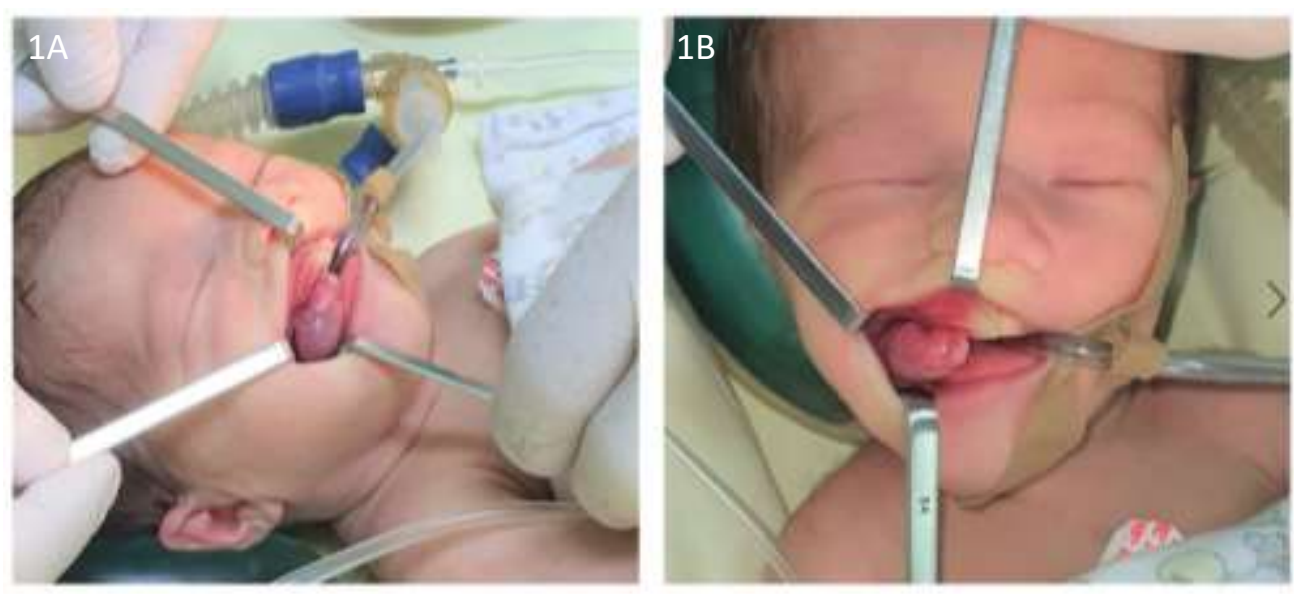

Fonte: Autores (2021).

A RN foi então encaminhada para avaliação com pediatra e radiologista do pronto socorro do Hospital UDI - São Luís/Maranhão, os quais suspeitaram de hemangioma e solicitaram para melhor investigação e início de terapia com betabloqueador os exames: tomografia computadorizada de face (TC), ultrassonografia transfontanela com doppler e eletrocardiograma (ECG). O pediatra relatou ainda, que a criança apresentava perda ponderal maior que $10 \%$ desde o momento do nascimento, constatando nutrição comprometida em função desta alteração de tecidos moles intraorais, sugerindo então, a cirurgia de remoção desta lesão. Segundo informado pelos responsáveis, a RN apresentou ainda episódios de refluxo com apneia, desta forma foi iniciado fórmula e manobras anti-refluxo.

Antes de qualquer terapia ser instituída, a referida paciente foi então encaminhada para avaliação com um especialista em Cirurgia e Traumatologia Buco-Maxilo-Facial com o intuito de um parecer mais assertivo da região acometida. Durante a análise de TC (Figuras 2A e B), notou-se lesão de aspecto nodular sem envolvimento ósseo, e, ao analisar a ultrassonografia transfontanela e ECG foi notado que todos os parâmetros estavam dentro dos padrões de normalidade. 
Figuras 2A e B - Tomografia computadorizada de face.

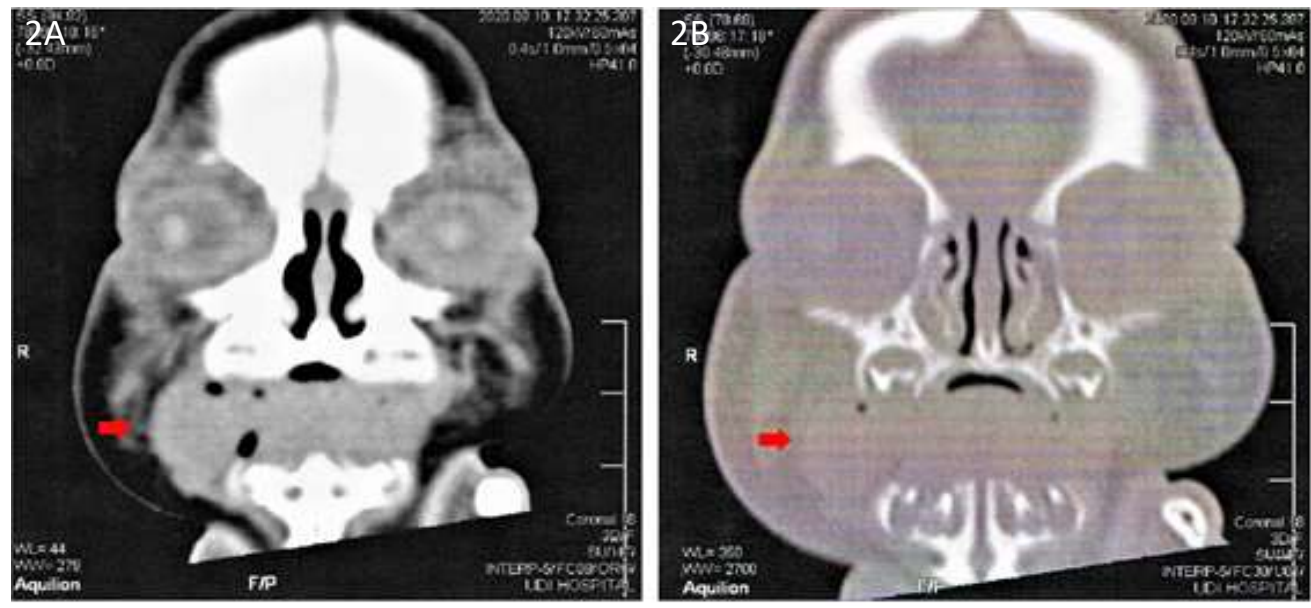

Fonte: Autores (2021)

O cirurgião buco-maxilo-facial ao avaliar o caso e todo histórico da paciente, solicitou exame de ultrassonografia com doppler da região de maxila lado direito para avaliação do fluxo sanguíneo da lesão e exclusão definitiva de hemangioma. Ao retornar para planejamento de procedimento cirúrgico e orientações finais, foi analisado o exame de ultrassonografia com doppler da região de maxila direita (Figuras 3A e B), no qual foi constatado que a lesão apresentava vascularização mínima periférica, descartando a hipótese diagnóstica anterior de hemangioma e sugerindo então a possibilidade de se tratar de epúlide congênita. Desta forma, foi solicitado avaliação pré-anestésica, a qual foi respondida como liberada para o procedimento proposto de exérese sob anestesia geral para maior conforto e segurança tanto para a RN quanto para a equipe cirúrgica.

Por se tratar de um caso conduzido de forma multidisciplinar, a paciente foi novamente encaminhada ao pediatra para avaliação final do compilado de exames e proposta cirúrgica, o qual, por fim, liberou a RN para procedimento, com internação em UTI neonatal previamente para controle glicêmico durante o jejum solicitado pelo anestesista para anestesia geral.

Figuras 3A e B - Ultrassonografia com doppler região de maxila direita.

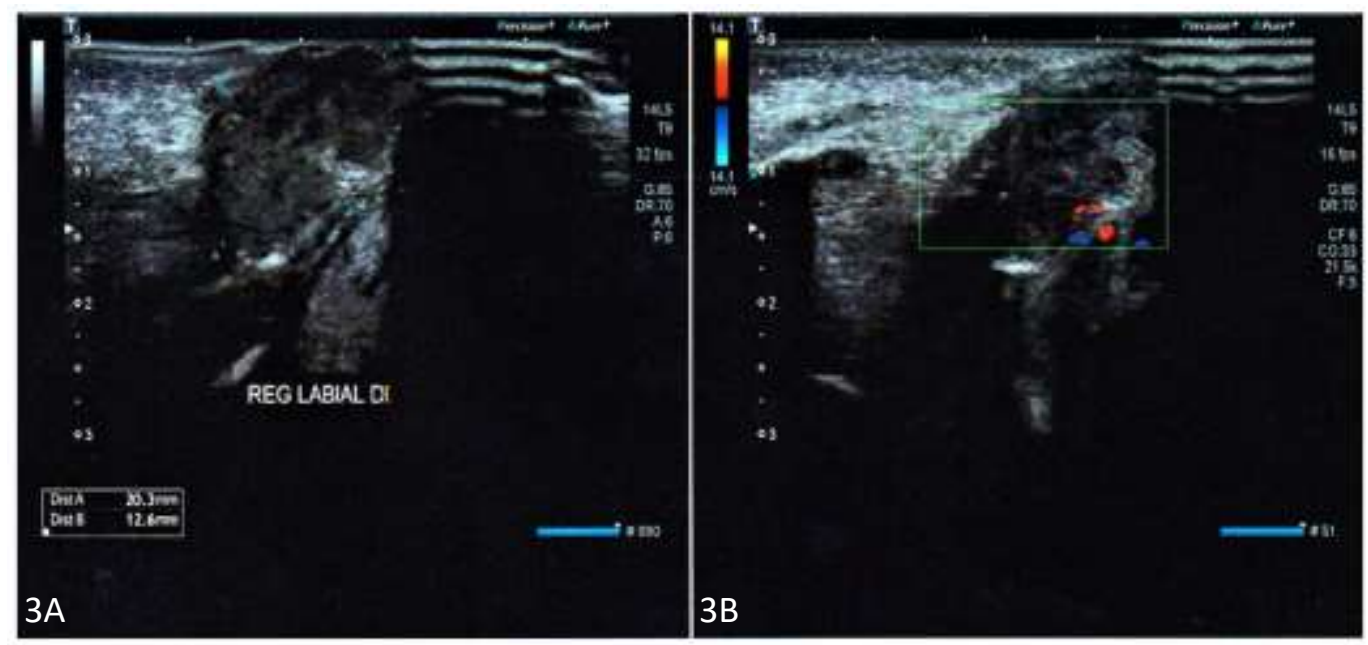

Fonte: Autores (2021).

A RN foi submetida ao procedimento cirúrgico sob anestesia geral no centro cirúrgico da Maternidade São Marcos em São Luís/Maranhão com 11 dias de vida, o qual foi devidamente realizado sem intercorrências através de excisão da lesão com bisturi frio e eletrocautério para a coagulação de pequenos vasos sangrantes, não sendo necessária a realização de suturas (Figuras 4A, B e C). A peça anatômica foi então acondicionada em formol 10\% e enviada para análise histopatológica. 
Figuras 4A, B e C - Transcirúrgico.
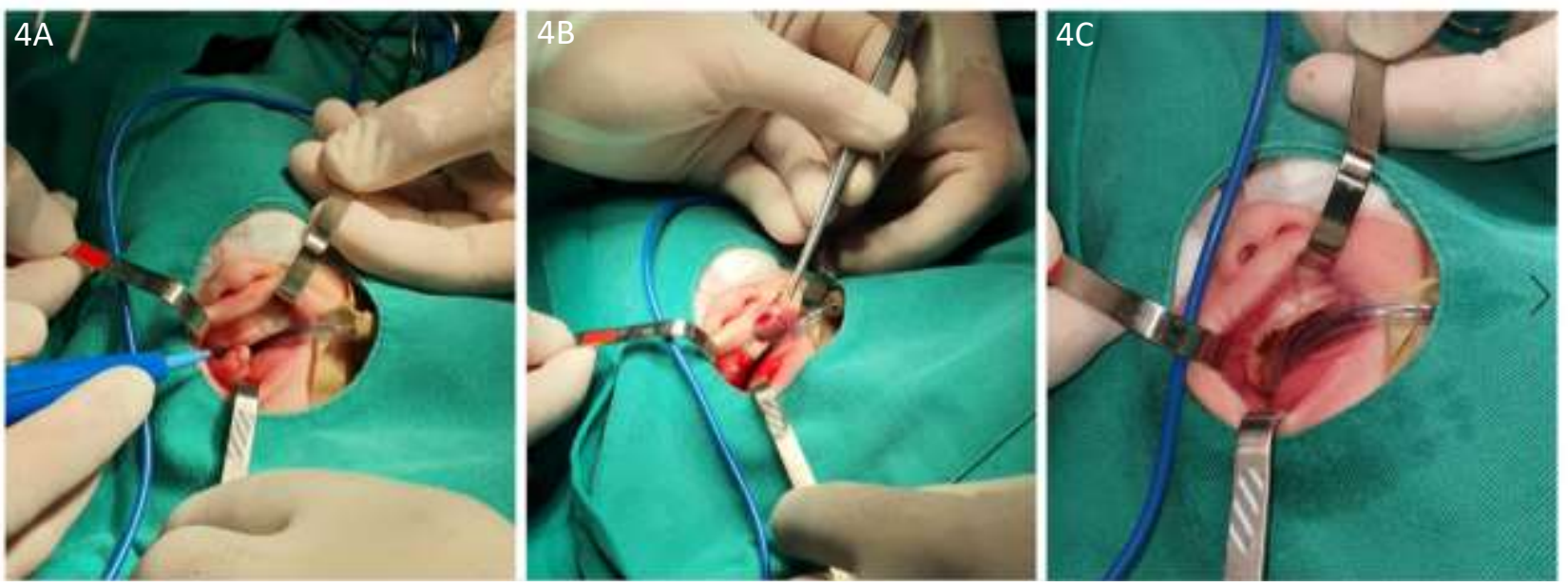

Fonte: Autores (2021).

Durante a análise histopatológica foi encontrada proliferação de células poligonais com citoplasma granular eosinofílico e núcleos excêntricos sem atípias citológicas, não se observando mitoses e/ou necrose (Figuras 5A e B). A análise imuno histoquímica não evidenciou marcação para a proteína S-100, confirmando por fim, o diagnóstico de epúlide congênita.

Figuras 5A e B - Lâmina histológica.
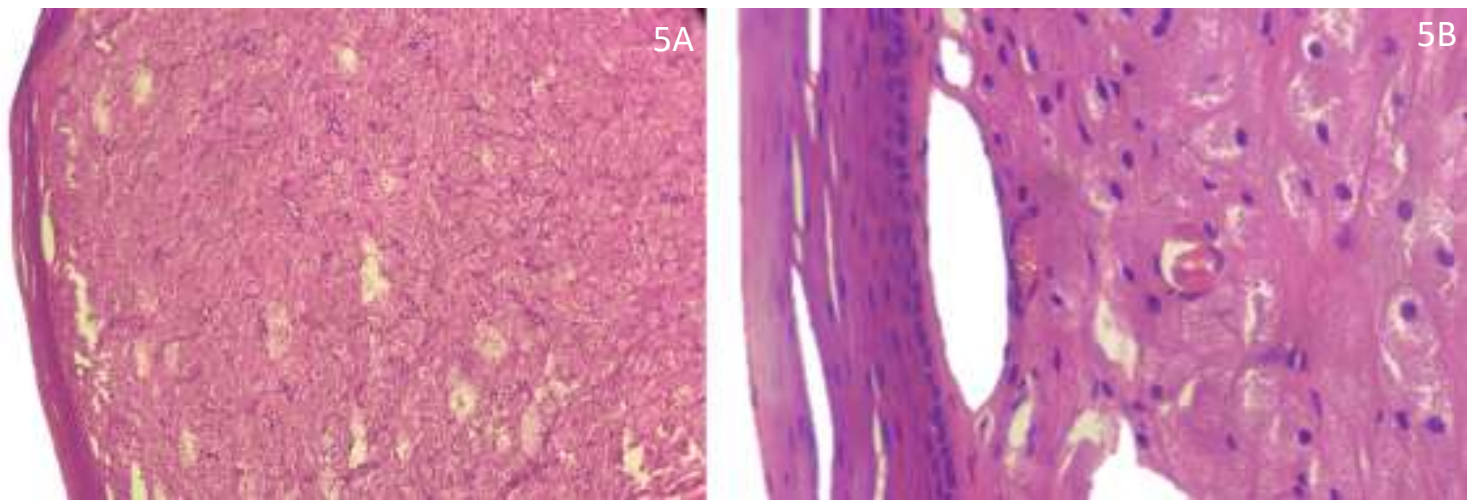

Fonte: Autores (2021).

Após a cirurgia, a instituição da nutrição foi imediata à liberação do anestesista e equipe neonatal, para a adequada recuperação nutricional, a qual foi possível sem irritabilidade ou dificuldades de pega à mama da mãe.

Após seis meses da realização do procedimento, foi identificado rebordo alveolar com aparência de normalidade, sem sinais de recorrências e nem dificuldades durante a alimentação, corroborando com a literatura sobre o sucesso da intervenção cirúrgica precoce nesses casos.

\section{Discussão}

A epúlide congênita ou tumor congênito de células granulares é uma lesão benigna, rara e encontrada apenas em fetos e recém-nascidos. A região anterior de maxila é acometida em uma razão de 3:1 quando comparada à mandíbula, assim como, os bebês do gênero feminino tem uma taxa de acometimento de até 10 vezes maior quando comparados aos de gênero 
masculino (Cheung \& Putra, 2020; Gómez, Carazo, Ballester, \& Sempere, 2019; Jain et al., 2020; Marinho et al., 2020; Rahman et al., 2015; Sasaki, Okamoto, Watanabe, Kagawa, \& Ando, 2018; Vinay, Anjulo, Nitin, Neha, \& Dhara, 2017). Existem autores que sugerem uma proporção de distribuição de 2:1 para maxila e identificação aumentada de 8:1 para indivíduos leucodermas do gênero feminino, corroborando com o exposto sobre a predileção pelo sítio maxilar e o menor número de pacientes do gênero masculino (de Paulo et al., 2013; Elías Podestá et al., 2017; Rodrigues et al., 2019; Wong, Ramli, Muhaizan, \& Primuharsa Putra, 2016).

O termo "Epulis" é oriundo de uma palavra grega que tem como significado "na gengiva" e segue sem alterações desde a sua última atualização em 2017 pela Organização Mundial da Saúde (OMS) (Jain et al., 2020; Rodrigues et al., 2019). Esta patologia foi primeiramente descrita por Neumann em 1871, que identificou inicialmente suas características, a sua baixa incidência, e a classificou como rara (Gómez et al., 2019; Jain et al., 2020; Lee et al., 2013; Vinay et al., 2017). Foram relatados na literatura menos de 100 casos de epúlide congênita (Cheung \& Putra, 2020; Lee et al., 2013).

Embora sua etiologia não seja bem esclarecida, ela possui relação direta com alterações hormonais gestacionais, se manifestando durante o terceiro trimestre e cessando seu desenvolvimento ao nascimento (Cheung \& Putra, 2020; Lee et al., 2013). Uma teoria sobre o estímulo hormonal endógeno foi sugerida para justificar a alta predominância para o gênero feminino, porém, sem comprovações (Jain et al., 2020).

Foi relatada a estabilização da epúlide congênita após o nascimento, o que sugere que a lesão tem íntima relação de seu desenvolvimento com os hormônios maternos durante a gravidez. Foi descrito ainda por alguns autores que esta lesão regrediu espontaneamente após o nascimento, embora a regressão espontânea possa ocorrer, isto não deve ser esperado, pois a epúlide congênita de dimensões importantes ou múltiplas, pode causar problemas de alimentação, respiratórios, comprometer a estética e o desenvolvimento das estruturas maxilofaciais (Rahman et al., 2015). No caso clínico relatado, a lesão interferia na pega da mama, causando irritabilidade e dificuldade nutricional, portanto, a remoção precoce foi necessária.

Esta lesão exofítica pediculada possui como características macroscópicas comuns: aspecto de massa nodular, firme à palpação e coloração rosada (Rahman et al., 2015). A sua histogênese não é bem definida, embora as células de Schwann, fibroblastos ou células mesenquimais tenham sido sugeridas como candidatas para a sua origem embriológica (Cheung \& Putra, 2020).

Esta é uma alteração isolada, sendo considerada "solitária", mas já foi relatado que pode ser identificada crescimentos múltiplos ou multilobulados. Não tem associação com síndromes, excluindo assim, a possibilidade de diagnósticos incorretos (Lee et al., 2013; Rahman et al., 2015). Não apresenta comportamento neoplásico, degenerativo ou reativo, o que confirma a sua não malignidade (Lee et al., 2013).

Durante a análise histológica, pode ser encontrado ninhos de células poligonais a discretamente fusiformes com citoplasma granular eosinofílico. Os núcleos estão excentricamente localizados com uma aparência geral branda. Estudos imuno-histoquímicos revelam intensa coloração para vimetina, STRO-1 e CD44, sugerindo que a sua origem tenha relação com a diferenciação dos tecidos mesenquimais (Cheung \& Putra, 2020; Kokubun et al., 2018; Wong et al., 2016).

Como se trata de uma lesão localizada na região anterior da boca, dependendo de suas dimensões, esta, pode dificultar a alimentação, fechamento da boca e respiração. O diagnóstico e tratamento precoces, como em várias outras situações, se tornam essenciais para que se evite o comprometimento nutricional, respiratório ou mesmo estético da criança. (Cheung \& Putra, 2020; Gómez et al., 2019; Johnson, Shainker, Estroff, \& Ralston, 2017; Rahman et al., 2015; Tiwari, Nagdeve, Saoji, Nama, \& Joshi, 2020).

O diagnóstico pode ser realizado durante a vida intrauterina através de exames de ultrassonografia (US) e de ressonância nuclear magnética (RNM), quando estas massas patológicas apresentam grande volume e se projetam para o exterior da boca do feto, porém, frequentemente é detectado apenas durante o nascimento. (Cheung \& Putra, 2020; Jain et al., 
2020; Rahman et al., 2015). A RNM pode ser indicada para diferenciar a epúlide de outras massas da cavidade oral, facilitando assim o planejamento cirúrgico adequado para esta situação (Gómez et al., 2019).

O ideal seria identificar a este crescimento tecidual no período pré-natal através de US, porém, esta possibilidade está intimamente relacionada ao tamanho da lesão e à capacidade técnica do operador do equipamento. O primeiro caso relatado na literatura através de US foi identificado na $26^{\mathrm{a}}$ semana de gestação, podendo muitas vezes ser detectado apenas ao nascimento durante o exame físico. A identificação precoce através de US permite o planejamento cirúrgico prévio ao nascimento e o preparo psicológico da família para a intervenção, porém, caso a identificação ocorra apenas durante o parto, é imprescindível a garantia, manutenção e proteção das vias aéreas antes de qualquer abordagem (Rahman et al., 2015).

Exames como US e RNM auxiliam na identificação da morfologia alterada na região oral durante a gestação, porém, as US pré-natais muitas vezes se mostram inespecíficas, assim, o diagnóstico diferencial deve sempre envolver hemangioma, encefalocele, cistos dermoides, malformações linfáticas, tumor neuroectodérmico da infância e rabdomiossarcoma quando nos deparamos com essas imagens (Jain et al., 2020).

As modalidades de tratamento para estas lesões podem ser diversas, desde acompanhamento clínico até excisões cirúrgicas. Sendo importante sempre as correlacionar com situações mais graves como miofibroma infantil, rabdomioma, rabdomiossarcoma, sarcoma osteogênico, fibroma odontogênico periférico, tumor neuroectodérmico melanótico da infância, e, neurofibroma, pois nesses casos a evolução pode ocasionar situações catastróficas (Cheung \& Putra, 2020; Rahman et al., 2015). Já foi relatado regressão espontânea, mas quando a lesão possui dimensões importantes, que impossibilitem a alimentação e respiração adequada, estas devem ser removidas de imediato (Lee et al., 2013).

Quando a lesão tem dimensões muito grandes ou interfere diretamente na alimentação ou respiração, o tratamento de escolha deve ser a remoção cirúrgica simples. As exéreses radicais ou com grandes margens de segurança não deverão ser realizadas, pois além da possibilidade de causar danos aos tecidos adjacentes, não foi relatado na literatura nenhuma recorrência ou degeneração maligna mesmo em casos de remoções parciais (Rahman et al., 2015).

A excisão do tumor não compromete o crescimento ósseo ou irrompimento dos dentes, sendo a massa tumoral é facilmente removida por ser pediculada na grande maioria dos casos (Jain et al., 2020; Lee et al., 2013). O exame de US com doppler da região se torna de grande valia após o nascimento para o diagnóstico e planejamento cirúrgico correto, pois avalia o crescimento vascular na região acometida e pode auxiliar na exclusão de outras lesões como por exemplo, o hemangioma, assim como foi realizado no caso exposto neste trabalho.

\section{Conclusão}

A epúlide congênita é um acometimento intraoral raro que pode ser identificado através de US durante o período gestacional ou apenas clinicamente durante o nascimento. Embora esta lesão tenha uma aparência impactante e angustiante, o seu curso e comportamento é benigno e não progressivo após o nascimento. Muitas vezes a abordagem cirúrgica precoce se torna necessária para evitar complicações maiores, como obstrução de vias aéreas, dificuldade nutricionais, comprometimento da oclusão da boca e estético do recém-nascido. Após o tratamento cirúrgico de remoção da lesão, é demonstrado uma evolução e prognóstico excelentes, sem associação com síndromes, alterações genéticas ou degenerações malignas.

\section{Referências}

Cheung, J. M., \& Putra, J. (2020). Congenital Granular Cell Epulis: Classic Presentation and Its Differential Diagnosis. Head Neck Pathol, 14(1), 208-211. $10.1007 / \mathrm{s} 12105-019-01025-1$

de Paulo, L. F. B., da Cruz Perez, D. E., Rosa, R. R., \& Durighetto Junior, A. F. (2013). Multiple congenital epulis: A case report with spontaneous regression. International Journal of Pediatric Otorhinolaryngology Extra, 8(3), 99-101. https://doi.org/10.1016/j.pedex.2013.06.003 
Research, Society and Development, v. 10, n. 2, e6910212324, 2021

(CC BY 4.0) | ISSN 2525-3409 | DOI: http://dx.doi.org/10.33448/rsd-v10i2.12324

Elías Podestá, M., Cóndor Astucuri, J., García Gutiérrez, M., Arellano Sacramento, C., \& Elías Díaz, P. (2017). Épulis del recién nacido \%J Pediatría Atención Primaria. 19, 275-278.

Gómez, A., Carazo, M., Ballester, J., \& Sempere, V. (2019). Épulis congénito gigante: importancia del diagnóstico prenatal. Anales de Pediatría, 93. 0.1016/j.anpedi.2019.06.008

Jain, N., Sinha, P., \& Singh, L. (2020). Large Congenital Epulis in a Newborn: Diagnosis and Management. Ear Nose Throat J, 99(7), Np79-np81. $10.1177 / 0145561319851498$

Johnson, K. M., Shainker, S. A., Estroff, J. A., \& Ralston, S. J. J. J. o. U. i. M. (2017). Prenatal Diagnosis of Congenital Epulis: Implications for Delivery. $36(2), 449-451$.

Kokubun, K., Matsuzaka, K., Akashi, Y., Sumi, M., Nakajima, K., Murakami, S., . . . Inoue, T. (2018). Congenital Epulis: A Case and Review of the Literature. Bull Tokyo Dent Coll, 59(2), 127-132. 10.2209/tdcpublication.2017-0028

Lee, J.-M., Kim, U.-K., \& Shin, S.-H. (2013). Multiple congenital epulis of the newborn: A case report and literature review. Journal of Pediatric Surgery Case Reports, 1(3), 32-33. https://doi.org/10.1016/j.epsc.2013.03.002

Marinho, L. C. N., Santos, H. B. P., Morais, E. F., \& Freitas, R. A. (2020). Benign neural lesions of the oral and maxillofacial complex: a 48-yearretrospective study \%J Jornal Brasileiro de Patologia e Medicina Laboratorial. 56.

Rahman, N., Ismail, H., Mohamad, I., Yusof, S., \& Mohamad, H. (2015). Congenital mandibular epulis - A rare oral lesion in a newborn. Egyptian Journal of Ear, Nose, Throat and Allied Sciences, 16, 295-298. 10.1016/j.ejenta.2015.07.003

Rodrigues, K. S., Barros, C. C. S., Rocha, O. K. M. S., Silva, L. A. B., Paies, M. B., \& Miguel, M. C. C. (2019). Congenital granular cell epulis: case report and differential diagnosis \%J Jornal Brasileiro de Patologia e Medicina Laboratorial. 55, 281-288.

Sasaki, R., Okamoto, T., Watanabe, Y., Kagawa, C., \& Ando, T. (2018). Congenital Granular Cell Epulis. Plastic \& Reconstructive Surgery Global Open, Latest Articles, 1. 10.1097/GOX.0000000000001989

Tiwari, C., Nagdeve, N., Saoji, R., Nama, N., \& Joshi, S. (2020). Large Congenital Epulis: A Neonatal Tumour with Striking Appearance, but Simple Management. Fetal and Pediatric Pathology, 1-3. 10.1080/15513815.2020.1805532

Vinay, K. N., Anjulo, L. A., Nitin, P., Neha, K. V., \& Dhara, D. (2017). Neumann's Tumor: A Case Report. Ethiopian journal of health sciences, 27(2), 189192. 10.4314/ejhs.v27i2.11

Wong, D. K., Ramli, R., Muhaizan, W. M., \& Primuharsa Putra, S. H. (2016). Congenital epulis: A rare benign tumour. Med J Malaysia, 71(5), 300-301. 